\title{
Estrategias de comunicación para impactar conductas (COMBI) en enfermedades vectoriales en América Latina
}

\author{
Andrea Jineth Rodriguez Reyes ${ }^{\mathrm{a}}$, Claudia Roncancio Melgarejo ${ }^{\mathrm{b}}$, Sandra Misnaza Castrillón ${ }^{\mathrm{c}}$ \\ aEspecialista en Epidemiología General. Instituto Nacional de Salud.andrea.jineth.rodriguez@gmail.com iD \\ https://orcid.org/0000-0001-5529-0293 \\ ${ }^{b}$ Magíster en Salud Pública. Secretaría Distrital de Integración Social cMagíster en Administración en Salud. Instituto Nacional de Salud \\ DOI 10.22517/25395203.18721
}

\section{Resumen}

Introducción: Las enfermedades transmitidas por vectores -ETV- están determinadas por una compleja dinámica de factores medioambientales y sociales, estrategias de control han encaminado históricamente conocimiento biológico sobre el vector. Experiencias mundiales muestran que el cambio del comportamiento de las personas es crucial para la prevención de estas enfermedades. La OMS, promovió la metodología COMBI (Communication for Behavioural Impact) para la planificación de acciones de comunicación y movilización social.

Objetivo: Compilar las experiencias de implementación de estrategia COMBI en América Latina para identificar fortalezas, amenazas y oportunidades de mejora de la metodología como herramienta para la mitigación de ETV.

Materiales y métodos: Revisión teórica tipo monografía, se incluyeron artículos y documentos indexados, documentos técnicos y literatura gris.

Resultados: La integración de la estrategia COMBI a los planes de salud pública permite la disminución de la frecuencia, magnitud y gravedad de los brotes y epidemias de las ETV en América Latina. Colombia es uno de los países que ha sistematizado las experiencias y los resultados de la implementación de la estrategia COMBI, lo que le permite a la región tener más argumentos para promover la estrategia.

Conclusiones: El cumplimiento de los objetivos conductuales, la integración de equipos multidisciplinarios, la investigación formativa, la movilización comunitaria y la abogacía son fortalezas que se evidencian en la implementación de la estrategia COMBI en América latina, y la falta de voluntad política y el cambio constante de personal inciden directamente en el éxito de la estrategia en la región.

Palabras clave: Salud pública, comunicación en salud, control de vectores, prevención, participación comunal.
Communication strategies for Behavioral Impact

(COMBI) in vector diseases in Latin America

\begin{abstract}
Introduction: Vector-borne diseases are determined by a complex dynamic of environmental and social factors; their control strategies have been historically directed by biological knowledge about the vector. Global experiences have shown that behavioral changes in people are a crucial element for the prevention of these diseases. The World Health Organization promoted the COMBI methodology (Communication for Behavioral Impact) to plan communication and social mobilization actions.
\end{abstract}

Objective: To compile the experiences about the implementation of the COMBI strategy in Latin America in order to identify the strengths, threats, and opportunities for improving this methodology as a tool for vector-borne disease mitigation.

Materials and methods: A theoretical-type monograph review was done. The materials included articles, index and technical documents, and grey literature.

Results: In Latin America, the integration of the COMBI strategy into public health plans allows the reduction of the frequency, magnitude, and severity of outbreaks and epidemics of vector-borne diseases. Colombia is one of the countries that has systematized the experiences and results of the implementation of the COMBI methodology, allowing the region to have more arguments to promote the strategy.

Conclusions: The accomplishment of the behavioral objectives, the integration of multidisciplinary teams, formative research, community mobilization, and advocacy are strengths that are evident in the implementation of the COMBI strategy in the Americas' region. On the other hand, the lack of political will and the constant change of personnel directly affect the success of the methodology in the territory.

Keywords: Public health, health communication, vector control, prevention, Community Participation. 


\section{Introducción}

La Organización Mundial de la Salud (OMS) establece que las enfermedades transmitidas por vectores están determinadas por una compleja dinámica de factores medioambientales y sociales y representan el $17 \%$ de todas las enfermedades infecciosas a nivel mundial, provocando más de un millón de defunciones anualmente. Dentro de estas enfermedades se describen el paludismo, dengue, esquistosomiasis, tripanosomiasis africana humana, leishmaniasis, enfermedad de Chagas, fiebre amarilla, encefalitis japonesa y oncocercosis $(1)^{\circ}$

Históricamente, las estrategias de control de vectores han aprovechado el conocimiento biológico sobre las especies de vectores para restringir el tamaño de sus poblaciones y limitar el contacto humano; estas han incluido la eliminación de los sitios de reproducción de vectores, mecanismo más antiguo de reducción de la población y la aplicación generalizada de insecticidas químicos ${ }_{(2)}$. La reducción del contacto huéspedvector también se ha implementado como una estrategia de control más reciente que utiliza combinaciones de barreras químicas (repelentes de insectos) y físicas (cobertura completa de ropa, redes de cama, pantallas de ventana) ${ }_{(3)}$. Sin embargo, experiencias mundiales han demostrado que, el cambio del comportamiento de las personas son un elemento crucial para la prevención de las enfermedades transmitidas por vectores y representan un desafío crítico que no puede ser abordado sin una mejor comprensión de cómo las personas perciben y reaccionan ante el riesgo de infección. Por esta razón, la OMS colabora con asociados con el fin de difundir conocimientos y mejorar la sensibilización, de manera que las personas sepan cómo protegerse a sí mismas y proteger a sus comunidades contra mosquitos, garrapatas, chinches, moscas y otros vectores.

En el 2000, la OMS promovió la metodología COMBI por sus siglas en inglés (Communication for Behavioural Impact) para la planificación de acciones de comunicación y movilización social. Esta estrategia de comunicación para impactar conductas es definida como un proceso que combina estratégicamente una variedad de intervenciones de comunicación destinadas a involucrar a individuos y familias en la adquisición de comportamientos saludables recomendados y alienta a la adopción y el mantenimiento de esos comportamientos $(4)$.

Los elementos esenciales de esta estrategia son: el establecimiento de objetivos conductuales precisos sobre la base de una investigación profunda, la integración de una mezcla sensata de acciones de comunicación pertinentes a los diversos grupos destinatarios y resultados conductuales deseados, todo cuidadosamente coordinado y cronometrado y el monitoreo constante del progreso hacia el logro de estos objetivos conductuales $_{(4)}$.

La metodología COMBI se diferencia de los enfoques tradicionales de información, educación y comunicación (IEC) porque permite avanzar en los programas más allá de la toma de conciencia para llegar al logro de objetivos conductuales precisos. Su metodología integra de manera efectiva la educación para la salud, la IEC, la movilización de la comunidad, las técnicas de comunicación del consumidor y la investigación de mercado, dirigidas de forma aguda e inteligente a resultados específicos y precisos de comportamiento en salud ${ }_{(4)}$.

En el plano de los programas de nueva generación, la metodología de planificación COMBI ha sido un componente central, convirtiéndose en un aspecto importante de los programas estratégicos en América Latina y como parte del compromiso de cooperación técnica con los países de la región, la Organización Panamericana de Salud (OPS), ha brindado apoyo a través de la capacitación en esta metodología, impulsando a su vez, la implementación de proyectos COMBI en Argentina, Brasil, Chile, Costa Rica, Colombia, Guatemala, Ecuador, El Salvador, México, Nicaragua, Paraguay, Perú, República Dominicana y Venezuela ${ }_{(5)}{ }^{\circ}$

El objetivo de esta revisión fue compilar las experiencias sobre la implementación de la estrategia COMBI en países de América Latina para identificar fortalezas, amenazas y oportunidades de mejora de la metodología como herramienta para la mitigación de enfermedades transmitidas por vectores en Colombia.

Se realizó una búsqueda de publicaciones en los que se hacía uso de la estrategia COMBI para la mitigación de enfermedades transmitidas por vectores en las bases de datos PubMed, ProQuest, LILACS, Medlinem, National Center for Biotechnology Information (NCBI), REPIDISCA y Scielo, usando las palabras clave: comunicación en salud, conductas relacionadas con la salud, control de vectores, participación de la comunidad, planificación social, planificación en salud comunitaria, investigación participativa basada en la comunidad y conductas de salud.Los criterios de selección de las publicaciones fueron: estudios publicados entre el 2002 al 2017 en los que se empleara la estrategia COMBI para la mitigación de enfermedades transmitidas por vectores en América Latina y que se encontraran publicadas en español, inglés y portugués. El análisis final incluyó 43 publicaciones relacionadas con en la implementación de la estrategia COMBI en Latinoamérica. 


\section{Definición de la estrategia COMBI}

La estrategia COMBI (Communication for Behavioral Impact), definida como la metodología de comunicación para impactar la conducta, es una estrategia para planificar la comunicación y conseguir la movilización social para lograr mantener resultados conductuales específicos relacionados con la prevención y el control de las enfermedades transmisibles ${ }_{(6)}$.

Esta estrategia inicia en 1994, en el instituto de verano de la Universidad de Nueva York, con un enfoque basado en la comunicación de marketing integrada, en el cual se desarrolla un programa de comunicación cuidadosamente planeado y monitoreado para involucrar a individuos, familias y comunidades a considerar acciones con respecto a comportamientos específicos; esta estrategia fue creada principalmente para abordar enfermedades transmisibles especialmente las transmitidas por vectores como el dengue ${ }_{(7)}$.

En el 2001, la OPS insta a los Estados miembros a la incorporación en los programas de prevención y control del dengue la participación comunitaria, la educación sanitaria y estrategias de comunicación social dirigidas a promover el cambio de conducta, teniendo en cuenta el aumento de la epidemia de dengue, la amenaza de reurbanización de la fiebre amarilla, la implementación de programas nacionales de control del dengue poco eficaces que se centran principalmente en el uso de insecticidas y manejo de situaciones de emergencia sin la adopción de medidas eficaces a largo plazo, acompañado de la descentralización de los servicios sanitarios, la infestación por el vector Aedes aegypti y la falta de abastecimiento de agua y gestión de residuos sólidos adecuados para la población ${ }_{(8)}$.

En 2002, durante la 55 Asamblea Mundial de la Salud, la OMS promueve la metodología COMBI debido a que se ha evidenciado que la disminución sostenida de las poblaciones de vectores depende en gran medida del compromiso de los gobiernos y de la participación de las comunidades, tanto a nivel de planificación de estrategias de intervención como de ejecución de medidas de control para prevenir la reproducción de Aedes aegypti ${ }_{(9)}$.

Entre 1996 y 2002 se observa un incremento en 3,5 veces el número de casos de dengue en América latina. Brasil, Colombia y Venezuela ocuparon en el 2002 los tres primeros lugares en números de casos de dengue reportados en el continente acumulando el 87,3\% del total de reportes ${ }_{(10)}$ ) Por esta razón, en el 2003 la OPS promueve la metodología COMBI como un componente integral del marco de referencia para la nueva generación de programas de prevención y control del dengue en Latinoamérica, creando la estrategia GT-Dengue, con enfoque multisectorial, intersectorial e interdisciplinario, permitiendo la evaluación y continuidad de las acciones, con recursos nacionales para lograr una estrategia nacional sostenible y en consecuencia, la reducción de las tasa de incidencia y letalidad del dengue ${ }_{(11)}$.

La metodología COMBI puede ser empleada en cualquier patología en la que los comportamientos humanos susceptibles de ser modificados incidan directamente en la prevención y control de las enfermedades, siendo usada en la prevención de enfermedades transmitidas por vectores, en la prevención de tuberculosis ${ }_{(12)}$, enfermedades de transmisión sexual ${ }_{(13,14,15)}$, disminución de la ingesta de sal ${ }_{(16)}$ y cáncer ${ }_{(17)}$, entre otras.

Esta estrategia consta de tres fases programáticas: planificación, ejecución y vigilancia-evaluación. Inicialmente, esta estrategia contaba con 15 pasos, pero con base en las experiencias de 14 países de América Latina con la metodología, se realizó una modificación en el número de pasos para la planificación de la estrategia COMBI quedando reducido a 10 pasos, con el fin de hacerlo más operativo y accesible para el manejo de equipos en todos los niveles ${ }_{(4,18)}$.

La metodología COMBI también puede ser usada durante la respuesta a un brote, permitiendo comprender rápidamente los desafíos del control del mismo desde la perspectiva de las comunidades en riesgo, asegurándose que la población tome las medidas apropiadas durante un brote, además de motivar a las personas, apoyar las actividades de control y a utilizar eficazmente los recursos humanos y financieros disponibles $(19)^{*}$

Esta estrategia se diferencia de la movilización social porque se interesa en lograr un consenso nacional y llevar a cabo un proceso educacional amplio, exigiendo el análisis de las estructuras sociales $_{(20)}$. Asimismo, agrega el elemento de movilización social al modelo de mercadeo social para garantizar que los productos, los conceptos o las innovaciones se difundan ampliamente a través de diversos canales y a su vez, se diferencia de los enfoques tradicionales de IEC, al hacer avanzar los programas más allá de la toma de conciencia para llegar al logro de objetivos conductuales precisos ${ }_{(16)}$.

\section{Componentes de la estrategia}

Una intervención exitosa de comunicación conductual y social requiere una combinación consecuente e integrada de cinco áreas de acción de comunicación, integradas en un plan estratégico de comunicación:

a. Abogacía/relaciones públicas/movilización administrativa: consiste en organizar la información en argumentos que serán comunicados a través de diversos canales interpersonales y medios de comunicación, con miras a ganar la aceptación y el compromiso del liderazgo político y social ${ }_{(18)}$. 
Es importante divulgar pruebas de los estudios que muestran la carga social y económica del dengue y demostrar la eficacia en función de los costos de sus intervenciones. La vigilancia y evaluación cuidadosa de los resultados conductuales ayudan enormemente a aportar pruebas para abogar por un mayor apoyo ${ }_{(18)}$.

b. Movilización de la comunidad: la comunidad se define como el medio social local y ejerce la influencia de tomar las decisiones y modalidades tradicionales para lograr el objetivo conductual deseado ${ }_{(18)}$. Con el fin de ganar aceptación y apoyo de la comunidad, se debe incluir el uso de la investigación participativa, reuniones de grupo, sesiones de asociaciones, actividades escolares, medios de prensa tradicionales, la música, el baile, los espectáculos en los caminos, presentación de dramas sobre la comunidad, volantes, carteles, folletos, videos y visitas a domicilio ${ }_{(18)}$.

c. Publicidad apropiada sostenida: es dar a conocer una conducta, producto o servicio entre los clientes actuales y potenciales. La publicidad debe proyectarse a la audiencia específica y basarse en la investigación formativa. Los métodos principales de publicidad incluyen: folletos o volantes, correo directo, mensajes de correo electrónico, revistas, boletines informativos, periódicos, carteles y tableros de avisos, anuncios de radio, venta telefónica, anuncios de televisión, páginas web. La publicidad necesita ser masiva, repetitiva, intensa y persistente (M-RIP) comprometiendo a personas a examinar los méritos de la conducta recomendada para llevarla a cabo $_{(18)}$.

d. Comunicación interpersonal: busca informar, educar y además persuadir a la acción, lo que puede aumentar una relación y crear un diálogo constante de interacción/participación con el público objetivo e influir para cambiar la conducta y así obtener un resultado. Las actividades de las relaciones públicas incluyen: artículos, editoriales y cartas a directores de periódicos y revistas, carpetas de prensa, comunicados de prensa o alertas de noticias, anuncios de servicios públicos, informes anuales, formación de redes, novedades, presentaciones, eventos y ofertas especiales $_{(18)}$.

e. Promoción en puntos de venta: es una acción de comunicación importante que permite resaltar fácilmente las medidas accesibles y disponibles para el control de vectores, el diagnóstico y tratamiento de fiebre ${ }_{(18)}$.

\section{Desarrollo de la estrategia}

El desarrollo de la estrategia COMBI involucra voluntades políticas, recursos económicos, talento humano, estrategias sociales y tiempo para que las comunidades a las que se acompaña puedan adoptar prácticas protectoras saludables y preventivas para la disminución del riesgo de enfermar. Por lo anterior, se han propuesto tres fases ${ }_{(21)}$ : a) fase de planificación; b) fase de ejecución y c) fase de vigilancia-evaluación o sostenibilidad. Las tres fases se encuentran articuladas entre sí, de tal modo que permiten la implementación de procesos que pueden sostenerse en el tiempo y son ajustables, de acuerdo con las características de la enfermedad y la disponibilidad de recursos.

\section{a. Fase de planificación}

Durante esta fase, se implementan pasos transversales que se desarrollan a lo largo del proceso, tales como la conformación de un equipo interdisciplinario, sistematización de la estrategia, diseño de una ruta de monitoreo y evaluación y fortalecimiento de las capacidades del personal.

En esta fase se planea y desarrolla la investigación de carácter etnográfico con enfoque de salud, lo que proporciona la información y datos necesarios para la elaboración del plan operativo. A su vez, este plan se estructura a partir de la construcción de los planes de comunicación y movilización social, los cuales constituyen la intervención en los territorios y las comunidades donde están reflejadas las cinco acciones integradas de COMBI: gestión interinstitucional, movilización comunitaria, publicidad apropiada y sostenida, comunicación interpersonal y promoción en puntos de venta. Parks W. y Lloyd L. ${ }_{(18)}$ en el 2004 plantearon 15 pasos para llevar a cabo la planificación de la estrategia COMBI, pero luego de un análisis y llegando a un consenso, se redujeron a 10 pasos en el año $2011_{(18)}$, los cuales son identificados a continuación: 
Figura 1. Pasos para la planificación de la estrategia COMBI

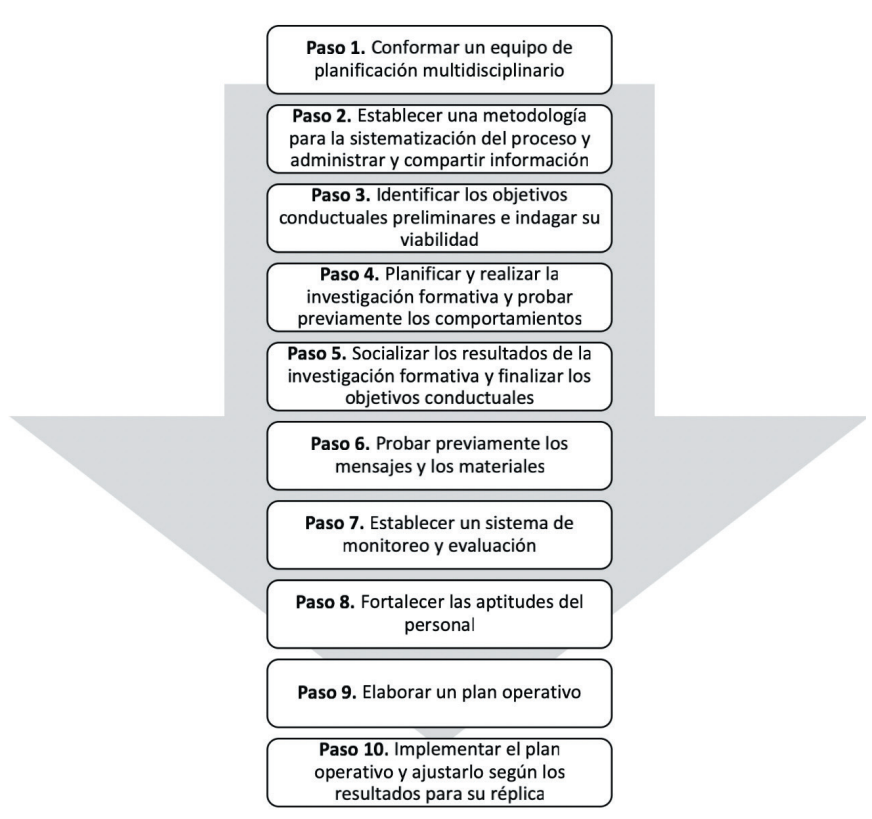

Fuente: Elaboración propia

En las enfermedades transmitidas por vectores (ETV), el equipo multidisciplinario debe estar conformado por profesionales de la salud, epidemiólogos, entomólogos, profesionales de las ciencias sociales, trabajadores de desarrollo comunitario, agentes de publicidad, planificadores urbanos e ingenieros ambientales, quienes aportan diferentes aptitudes y puntos de vista, de tal manera que el trabajo en equipo sea creativo y eficaz $_{(5,18)}$.

La metodología para sistematizar los procesos y administrar y compartir información se realiza con el fin de analizar los factores metodológicos que están contribuyendo u obstaculizando la consecución de las metas planteadas ${ }_{(19)}$. Es necesario analizar la estrategia a usar para compartir la información con grupos objeto como, por ejemplo, artículos de periódico, boletines por radio o representaciones visuales $\mathrm{y}$ folletos sencillos.

La prevención y el control de las enfermedades transmitidas por vectores dependen del logro de resultados conductuales específicos, por lo que el establecimiento de objetivos conductuales preliminares permite limitar la investigación, pensar en las necesidades futuras y dar forma inicial a las estrategias. De igual forma, estos objetivos pueden no ser factibles desde el punto de vista de los grupos que se espera influenciar, por lo que es posible usar la investigación formativa para indagar si las expectativas son razonables ${ }_{(5,18)}$.
Con respecto a la investigación formativa, esta se realiza principalmente al comienzo del programa e incluye toda la investigación que ayuda a informar el desarrollo de una nueva estrategia de movilización y comunicación social o el perfeccionamiento de una existente, basándose en los objetivos conductuales preliminares y permitiendo identificar los temas sociales clave, las necesidades de las comunidades y oportunidades para el cambio, las analogías culturales clave que pueden usarse para los mensajes de educación sanitaria, entre otros ${ }_{(5,18,22)}$. Por lo anterior, durante esta fase se propone realizar preguntas de investigación enmarcadas en las siguientes temáticas: comunicación, movilización social, acceso a servicios de salud, epidemiología, entomologíacontrol vectorial y sostenibilidad ${ }_{(21)}$.

La investigación formativa es usada también en la evaluación de la estrategia COMBI examinando las debilidades, oportunidades, fortalezas y amenazas (DOFA), con el fin de descubrir las necesidades de la comunidad que no están siendo satisfechas, no por causa de una movilización y comunicación social ineficaz, sino a causa de limitaciones de recursos significativos $_{(5,18)}$.

Es necesario divulgar los resultados de la investigación formativa con el fin de ser usados en diferentes secciones del programa, como gestión, logística, monitoreo, control de vectores, comunicaciones y adiestramiento; asimismo, compartir los resultados permitirá a los interesados directos conocer claramente las recomendaciones que justifican el cambio de conducta para disminuir las enfermedades transmitidas por vectores ${ }_{(5,18)}$.

Una de las características de una estrategia de movilización y comunicación social bien diseñada es la realización de pruebas de los mensajes y materiales, por lo que durante el proceso de planificación es importante evaluar cómo el público destinatario reacciona frente a las conductas, los mensajes y materiales prototipos propuestos, con el fin de identificar la necesidad de cambio antes de la ejecución o producción final. Con respecto a los métodos de control de Aedes, es importante este paso para determinar la probabilidad de que un producto nuevo sea aceptado, igualmente, permite evaluar si los mensajes son claros y convincentes, identificar los mensajes no intencionales, detectar las respuestas del público que son completamente impredecibles y seleccionar entre una variedad de mensajes y materiales posibles ${ }_{(5,18)}{ }^{\circ}$

Para la implementación exitosa de la estrategia COMBI es vital un sistema funcional de monitoreo y evaluación, este componente guía la planificación e implementación de la estrategia global, evalúa su efectividad, identifica áreas de mejora en los objetivos, público destinatario, conductas, productos, materiales, mensajes, canales de comunicación, 
redes de distribución, entre otros, y optimiza el uso de los $\operatorname{recursos}_{(23)}$.

En el proceso de seguimiento es necesario la presencia de expertos en ciencias sociales que apoyen el diseño del sistema de monitoreo así como los métodos de recolección y análisis de datos. Existen dos mecanismos para vigilar el progreso de la estrategia, el primero es mediante el monitoreo de los impactos conductuales y el segundo, mediante la evaluación del proceso ${ }_{(5,18)}$.

Las conductas específicas que pueden monitorearse incluyen: las prácticas de almacenamiento del agua; la adquisición, el almacenamiento y el desecho de recipientes, tales como neumáticos y latas; las prácticas encaminadas a prevenir las picaduras de mosquitos, tales como el uso de insecticidas y repelentes; y el tratamiento de casos de enfermedades febriles $(5,18)^{*}$

Por otro lado, es necesario fortalecer las aptitudes del personal, principalmente cuando las estrategias COMBI han sido descentralizadas o están en ese proceso, con el fin de brindar oportunidades al personal de servicio de aprender a desarrollar y ejecutar las estrategias de movilización y comunicación social adecuadas que permitan resolver el cómo escuchar a los miembros de la comunidad y trabajar con ellos y cómo vincular sus planes y actividades con las percepciones, condiciones y recursos locales. Además, se deben organizar cursos de formación en comunicación interpersonal, entre otros, para los voluntarios y el personal de otros organismos que apoyan la estrategia ${ }_{(5,18)}$.

La elaboración del plan operativo es el registro de los objetivos y la herramienta más importante para dirigir la puesta en práctica de las estrategias de movilización y comunicación social. La finalidad de este plan es que sea apropiado para el problema de salud y los grupos destinatarios identificados, aproveche los recursos disponibles y proporcione un impacto conductual duradero. Este documento puede consultarse y modificarse según sea necesario y, debe ser discutido y debatido por el equipo de planificación multidisciplinario y otros interesados directos ${ }_{(5,18)}$.

Los planes operativos pueden ser a corto plazo (un año o menos) o a largo plazo (entre tres a cinco años) y deben especificar de manera clara y exhaustiva los pasos que se seguirán para poner en práctica una estrategia o el conjunto de estrategias, con el fin de lograr sus objetivos. El plan debe incluir todas las actividades preparatorias e indicar lo que sucederá una vez que cada estrategia se haya puesto en práctica ${ }_{(5,18)}$.

El plan operativo debe incluir tres secciones básicas: introducción, enfoque estratégico y un plan de ejecución. La introducción contiene los principales resultados de la investigación formativa, los los objetivos conductuales y una descripción de los grupos destinatarios. El enfoque estratégico debe contener la meta general, los objetivos conductuales y un resumen general de la estrategia de movilización y comunicación social. Por su parte, el plan de ejecución debe contener acciones de comunicación, el monitoreo conductual y la evaluación de procesos a ser usados, una descripción del equipo de gestión, un plan de trabajo detallado que incluya el calendario de las actividades de preparación y ejecución necesarias para poner en práctica cada acción de comunicación y una lista detallada de los costos para las diversas actividades descritas en el plan de trabajo ${ }_{(5,18)}$.

Una vez elaborado el plan operativo, se debe realizar una prueba piloto con el fin de obtener retroalimentación de los participantes y del personal implicado en la puesta en práctica del plan sobre la calidad de las actividades en todas sus dimensiones, desde los materiales didácticos hasta la conveniencia del personal elegido para llevar a cabo dichas $\operatorname{actividades}_{(5,18)}$.

La prueba piloto y la introducción gradual de intervenciones con un monitoreo constante ayudan a evitar inversiones importantes en actividades ineficaces. Durante esta prueba se debe asegurar que cualquier cambio que se observa es el resultado de la estrategia y no de otros acontecimientos que están ocurriendo en el grupo destinatario, por lo que se debe incluir siempre un grupo testigo o control para el cual sólo se están realizando tareas habituales ${ }_{(5,18)}$.

Durante un brote epidémico también se puede implementar la estrategia COMBI, donde la la fase de planificación está constituida por los siguientes pasos: ${ }_{(19)}$

a. Identificar los objetivos conductuales preliminares

b. Realizar un análisis rápido del mercado situacional

c. Refinar los objetivos conductuales, establecer los objetivos de comunicación

d. Diseñar una estrategia global

e. Preparar planes de ejecución y monitoreo y presupuesto

f. Implementar y monitorear la estrategia, identificar tendencias y adaptarse si es necesario

g. Evaluar una vez que el brote ha terminado

\section{a. Fase de ejecución}

La fase de ejecución o intervención contempla la puesta en marcha del plan operativo construido en la fase de 
planificación. Una vez se ha realizado la prueba piloto y el plan se ha ajustado de acuerdo con la realidad del territorio y la recepción que en primera instancia tenga la comunidad, se procede a implementar el plan operativo y sus componentes de comunicación y movilización social ${ }_{(21)}$.

El éxito en la implementación del plan operativo depende de muchos factores, entre ellos están los siguientes ${ }_{(21)}$ :

i. Tener aliados estratégicos, tales como los Agentes Comunitarios en Salud (ACS), conocidos también como agentes de atención primaria o promotores de salud, quienes son líderes locales de salud que conocen sus comunidades y son reconocidos por ellas y se capacitan para apoyar los procesos COMBI. Esto facilitará desarrollar de manera sostenida y con gran impacto el plan operativo.

ii. Conformación de Comisiones Locales de Salud o COVECOM. Las comisiones están conformadas por habitantes locales que están siempre dispuestos a mejorar la calidad de vida de sus comunidades y son los encargados de apoyar y desarrollar todas las actividades que requieren de la participación de la comunidad, así como de ser replicadores de los conocimientos adquiridos sobre la enfermedad. Son cruciales para la puesta en marcha del plan operativo.

iii. Capacitación sobre el plan operativo al equipo interdisciplinario, los ACS y las Comisiones Locales de Salud de tal forma que se puedan generar sinergias con las demás acciones del programa de ETV.

iv. Despliegue de la prueba piloto, que sirve como entrenamiento del personal involucrado con la implementación de COMBI, con especial énfasisen las personasquetrabajarándirectamenteconlacomunidad.

\section{b. Fase de vigilancia-evaluación o sostenibilidad}

La fase de vigilancia-evaluación comienza a trabajarse desde el inicio de implementación de COMBI, pero se ubica hacia el final del desarrollo metodológico, con el fin de reforzar y potenciar la capacidad instalada tanto a nivel comunitario como institucional, apuntando a que los procesos tengan continuidad en el tiempo ${ }_{(19)}$.

Los programas de prevención y control de las ETV pueden implementar las tres fases de acuerdo con su disponibilidad de recursos, pero es recomendable no exceder los dos meses entre cada fase, ya que las dinámicas de los territorios siempre son cambiantes, lo que implicaría volver a realizar determinadas acciones, generando sobrecostos ${ }_{(21)}$.

Finalmente, se debe enfatizar que el objetivo de la estrategia
COMBI es el impacto en conductas y no solamente el aumento del conocimiento de la enfermedad por parte de las comunidades. En este sentido, se trata de acciones concretas que las personas pueden realizar para la prevención de la enfermedad, las cuales deben ser medibles y sostenibles en el tiempo $_{(21)^{\circ}}$

\section{Principales metodologías de la estrategia}

Durante la fase de planeación e implementación de la estrategia COMBI se usan diferentes metodologías para la adopción de comportamientos conductuales por parte de la comunidad.

En la India, se han realizado trabajos con artistas para la creación de canciones, dramas y obras de teatro musicales mediante los cuales se informa sobre los signos y síntomas, tratamiento, transmisión, proceso de atención, rol del vector y manejo ambiental e implementación de medidas de higiene en el hogar, con previa autorización de la comunidad y autoridades locales ${ }_{(24)}$.

En Colombia, se han diseñado planes de capacitación diferencial para cada grupo de equipos (técnicos y madres comunitarias) para medir el nivel de conocimiento de los participantes mediante preguntas y ejercicios de interlocución ${ }_{(25)}$. Asimismo, en el departamento de Sucre, durante la fase de planeación en implementación de la estrategia COMBI, se han usado técnicas de conversatorios, lluvia de ideas, talleres demostrativos de lavado de recipientes recolectores de agua, de reflexión crítica apoyado con ayudas educativas: folletos, plegables, láminas, bocetos, mensajes educativos, juegos y programa radial ${ }_{(26)}$.

En México, las metodologías usadas para la implementación de la estrategia COMBI fueron reuniones con la comunidad, producción de materiales de comunicación y educación a domicilio, asimismo, campañas de comunicación educacional, difundida a través de canales de comunicación personales (visitas a domicilio y actividades escolares). y canales de comunicación impersonales (radio y televisión) usando humor y expresiones locales ${ }_{(18)}$.

\section{Participación social}

La participación social -en la cogestión de la salud- se define como la acción de actores sociales con capacidad, habilidad y oportunidad para identificar problemas, necesidades, definir prioridades, formular y negociar propuestas en pro del desarrollo de la salud, de una manera deliberada, democrática y concertada ${ }_{0}$. A su vez, esta participación debe ser informada, activa, consciente, voluntaria, responsable e integral, por lo que es necesario que esté apoyado en programas y estrategias de educación y comunicación en salud ${ }_{(28)}$. 
En los últimos años, con el fin de alcanzar un mayor nivel de desarrollo de la participación social se ha vinculado el empoderamiento o empowerment, palabra que hace referencia a la capacidad de las personas de llevar a cabo acciones de forma individual o colectiva para alcanzar las metas propuestas $(29)^{\circ}$

La OMS, define al empoderamiento en salud como un proceso social, cultural, psicológico y político, mediante el cual los individuos y los grupos sociales son capaces de expresar sus necesidades, plantear sus preocupaciones, diseñar estrategias de participación en la toma de decisiones y llevar a cabo acciones políticas, sociales y culturales para hacer frente a las necesidades identificadas. En este sentido, los individuos actúan de manera colectiva con el fin de conseguir una mayor influencia y control sobre los determinantes de la salud y la calidad de vida de su comunidad ${ }_{(30)}$.

Con el fin de conseguir un empoderamiento de la población local, es necesario un proceso intenso de educación popular, involucrar a las propias poblaciones en proyectos de investigación -acción- participativa para disponer de diagnósticos reales que contengan la perspectiva de los interesados, la planificación de conjunto y la evaluación participativa. Otra de las estrategias es crear redes y alianzas que faciliten la negociación y solución de conflictos, así como que potencialicen la intersectorialidad ${ }_{(31)}$.

En el componente de Promoción de la Salud una de sus prioridades ha sido el empoderamiento (,) con el fin de que los individuos y las poblaciones controlen su salud mediante un abordaje multidimensional (ambiente, economía, cultura, política, etc.), en el cual las acciones para el cambio de comportamiento se dirijan al nivel individual y colectivo, a la toma de decisiones a nivel político, a los funcionarios facilitadores y ejecutores de las estrategias y a las comunidades receptoras de la misma ${ }_{(34,35)}$.

Se ha evidenciado que la participación comunitaria es vital para prevenir y controlar la propagación de las ETV $(18.36)$. En México, se han creado estrategias que incorporan la movilización social y el cambio de comportamiento a nivel comunitario con el objeto de mejorar la eficacia, la rentabilidad, el impacto ambiental y la sostenibilidad de las estrategias de lucha antivectorial, en las que se observa que el empoderamiento de la comunidad es un aspecto clave que permite a la población local lograr la eliminación de los factores de riesgo que inciden en la aparición de la enfermedad en su entorno, asimismo, se destaca que la participación social es necesaria en una serie de etapas de la estrategia de control de vectores como lo es en la evaluación de los problemas y necesidades de la comunidad, actividades de ejecución y en la evaluación de la estrategia ${ }_{(37)}$.
De igual forma, una investigación realizada en México y Nicaragua con el fin de evaluar la eficacia de la movilización comunitaria en el control del dengue, demostró que la estrategia basada en la evidencia puede agregar eficacia al control vectorial. A su vez, demuestra que la intervención implementada de acuerdo con la realidad local permite hacer más fuerte el compromiso de la comunidad en la ejecución ${ }_{(38)}$.

En Cuba, se demostró que la participación comunitaria en las actividades de prevención de las ETV como el dengue con un enfoque de empoderamiento, favorecen el cambio conductual de la comunidad y son efectivas en la reducción de los índices entomológicos ya que la combinación del empoderamiento de la comunidad con la coordinación intersectorial permiten el mantenimiento de los resultados después de que no exista apoyo externo ${ }_{(39)}$.

En Honduras entre 1990 y 1996 se implementó un modelo de movilización social con un enfoque más participativo centrado en la familia y en la comunidad, que evidenció los beneficios de la participación comunitaria en el diseño y promoción de comportamientos técnicamente efectivos y socialmente aceptables para mejorar el manejo de los embalses de agua ${ }_{(40)}$.

De igual forma, un estudio de evaluación en el que se compara la participación comunitaria y la gestión ambiental con un programa vertical de control entre el año 2000 al 2004 en Santiago de Cuba, evidencia que los costos económicos fueron similares pero que el costo social asociado a la reducción de focos reproductores de Aedes fue menor en el área de intervención (programa con participación comunitaria) que en el área de control (programa vertical) ${ }_{(41)}$.

Igualmente, se ha demostrado que la integración de organizaciones comunitarias a los programas verticales de control de vectores pueden conducir a un control sostenible y rentable de las ETV ${ }_{(42,43)}$ y también generan cambios a nivel macro-social para contribuir al desarrollo social ${ }_{(44)}$.

Finalmente, los programas para el control de vectores y la prevención de enfermedades como el dengue se consideran exitosos si alcanzan una amplia audiencia, son eficaces en condiciones ideales, son adoptadas por la mayoría de la comunidad, tienen un efecto confirmado en el comportamiento humano relacionado con el control del vector y pueden mantenerse durante un tiempo suficientemente $\operatorname{largo}{ }_{(45)}$.

Por lo anterior, el enfoque de participación social en la estrategia COMBI debe contemplar el uso de métodos de toma de decisiones democráticos y consensuados que pueden aumentar la satisfacción de los miembros de la comunidad, ampliar la participación y mejorar el mantenimiento de los efectos de la estrategia. 


\section{Impacto de la estrategia en América Latina}

Con el objetivo de dar respuesta al problema de las enfermedades transmitidas por vectores -principalmente el relacionado con el dengue-, la OPS/OMS impulsó en América Latina la Estrategia de Gestión Integrada para la prevención y control de esta enfermedad, en la que se incluye la metodología COMBI en el componente de comunicación y movilización social con el fin de lograr cambios de conductas sostenibles garantizando que los programas que tienen generalmente presupuestos y recursos humanos muy reducidos, obtengan una optimización de los recursos, en cuanto a los resultados conductuales reales que se esperan lograr ${ }_{(5)}$.

En Brasil se ha reemplazado el tradicional Programa de Erradicación del Aedes aegypti (PEA), por una EGI-dengue nacional que incluye la metodología COMBI, logrando un trabajo extrasectorial mediante una excelente coordinación entre la industria privada (la Asociación Nacional de la Industria del Neumático y su sindicato), la Secretaría de Calidad del Ambiente en los Asentamientos Humanos del Ministerio de Medio Ambiente y la propia comunidad, que permitió la creación de una red de centros de recepción de neumáticos inservibles denominados Ecopuntos y plantas de resección y corte, ya sea para usarlos como combustible, laminarlos para fabricar zapatos o para convertirlos en asfalto, reciclado a la fecha más de 70 millones de neumáticos ${ }_{(46)}$.

En Chile, la estrategia COMBI se quiso implementar en Isla de Pascua pero por razones administrativas como la falta de personal para la implementación del proyecto se detuvo su aplicación, lo que deja como lección que debe haber voluntad política del país, que permita apoyar con recursos económicos y humanos la zona donde se implemente la estrategia ${ }_{(5)}$.

En Punta Arenas , Costa Rica, mediante la implementación de la EGI-dengue se desarrolló un plan COMBI con resultados que reflejan cambios conductuales positivos en la población y un notable avance en la capacidad de respuesta ante los brotes de dengue ${ }_{(42)}$. Asimismo, en la ciudad de San José, desde el año 2006, se implementó de manera favorable la estrategia COMBI siendo el principal objetivo conductual la recolección de llantas en desuso, aunque se evidenciaron algunos inconvenientes como la renovación permanente de personal obstaculizando el desarrollo oportuno de las acciones ${ }_{(5)}$.

En Colombia, a través del proyecto Malaria Colombia se implementó la estrategia COMBI en los departamentos de Antioquia, Cauca, Chocó, Córdoba, y Valle del Cauca, en articulación y como fortalecimiento a los programas de promoción, prevención y control de las Enfermedades Transmitidas por Vectores (ETV) en cada uno de estos departamentos; durante este proceso se destacó que el uso del toldillo fue un objetivo viable, alcanzable y sostenido en el transcurso de la planeación e implementación de la metodología COMBI malaria, y que la sistematización de la experiencia, el seguimiento al proceso y el monitoreo al objetivo conductual permitió un mayor impacto de la intervención ${ }_{(47)}$.

En el departamento de Atlántico, Colombia, se usó la estrategia COMBI con el propósito de mejorar la efectividad y sostenibilidad de los programas de control de dengue, en esta investigación se da una gran importancia a las variables de carácter psicosocial, que actúan como predictores del comportamiento y muestran, de forma significativa, el tipo de decisiones que adoptan las personas con respecto a la enfermedad y los sitios de cría del vector ${ }_{(48)}$.

En el municipio de Sincelejo, mediante la estrategia COMBI se evaluó la eficacia de la intervención educativa para inducir cambios en la conducta y erradicación de criaderos, logrando cambios positivos en el porcentaje de niveles de conocimiento inadecuados y de prácticas adecuadas, y asimismo, en la reducción de criaderos intradomiciliarios de Aedes aegypti ${ }_{(49)}$.

Colombia ha sido uno de los países que le apostó al uso de COMBI para la prevención y control de la malaria, otras enfermedades transmitidas por vectores (ETV) y eventos de importancia en salud pública, lo cual le ha permitido sistematizar sus aprendizajes permitiendo que el país y la región cuenten con más elementos para hacerle frente a las ETV $_{(46)}$.

Además, en Colombia se han creado espacios de divulgación de los resultados de las estrategias COMBI implementadas en algunos departamentos de Colombia encontrándose que, en Santander, hubo inconvenientes políticos y de planificación que afectaron la implementación de la estrategia; en San Andrés se logró realizar una investigación formativa con enfoque etnográfico, las familias incorporaron a su cotidianidad las conductas saludables trabajadas y la estratificación de riesgo de índice aédico disminuyó. En Antioquia se logró implementar la estrategia COMBI con éxito, aunque se presentaron inconvenientes puesto que pese a los esfuerzos por generar comprensión sobre el problema en la comunidad, las personas siguieron con creencias que les hacían asociar cualquier tipo de síntoma con el dengue ${ }_{(50)}$.

En Ecuador, se ha implementado la estrategia COMBI integrando en la planificación y ejecución a estudiantes de promoción para salud y teniendo como objetivo conductual "que el 100\% de las amas de casa de la Cooperativa Guayas y Quil II - Guasmo Sur, laven y cepillen una vez por semana los tanque bajos que contienen agua y siempre los mantengan tapados adecuadamente" ${ }_{(51)}$. Al final de la implementación de esta estrategia, los resultados fueron satisfactorios, lográndose ejecutar en un $100 \%$ las estrategias de relaciones 
públicas / abogacía / movilización administrativa, en un $90 \%$ la movilización de la comunidad, en un $80 \%$ la publicidad apropiada sostenida, en el 75\% la comunicación interpersonal, en el $50 \%$ en la promoción de puntos de venta y en un $80 \%$ la vigilancia y evaluación de la estrategia ${ }_{(51)}$.

En 2006, en la ciudad de Santa Cruz de Galápagos , Ecuador, se implementó la estrategia COMBI con apoyo financiero de la OPS y el Centro de Control y prevención de enfermedades (CDC), con algunos inconvenientes que influyeron en que el proyecto no culminara con éxito, como un retraso de 10 meses en la fase de planificación por circunstancias políticas y un proceso de capacitación pero no de apropiación o empoderamiento de la estrategia COMBI por parte de los estudiantes y coordinadores de colegio incluidos en la estrategia, por lo que en terreno no hubo un personal bien capacitado que permitiera la implementación de la misma ${ }_{(5)}$.

Asimismo, en El Salvador, mediante la implementación de un plan COMBI, se han obtenido experiencias exitosas como lo es la disposición adecuada de neumáticos desechados en el municipio de San Martín ${ }_{(46)}$.

En Guatemala, se ha extendido la estrategia de gestión integrada EGI-dengue a departamentos y municipios, lo que ha facilitado las negociaciones del Ministerio de Salud con los gobiernos municipales y las organizaciones no gubernamentales (ONG) y comunitarias para el desarrollo de un proyecto COMBI, mejorando el control del dengue en los niveles intermedios y las acciones de prevención, vigilancia y alerta epidemiológica ${ }_{(48)}$. En el departamento de Zacapa, también se implementó con éxito la estrategia COMBI, aunque tuvo que superar inconvenientes con la comunidad como la apatía y la limitada participación debido a que no se les involucró desde un inicio $(5)^{\circ}$

En la ciudad de Guerrero, México, se usó la estrategia COMBI con el fin conseguir viviendas con patios limpios y libres de sitios de reproducción de Aedes aegypti con resultados favorables, $58 \%$ de hogares "limpios" y libres de sitios de reproducción; además, se observó que los hogares en los que no se implementó la estrategia tenían 2,4 veces mayor riesgo de desarrollar dengue ${ }_{(37)}$.

En Nicaragua, mediante la implementación de la estrategia COMBI se mejoró el monitoreo del uso de la cartilla de dengue, utilizada en las escuelas primarias por orientación del Ministerio de Educación; asimismo, en los barrios donde se implementó el plan COMBI se crearon espacios de participación que antes no existían, integrando a las iglesias y los gobiernos municipales, y se ha constatado la disminución de los niveles de transmisión de dengue en los últimos dos años ${ }_{(46)}$.
Durante los años 2007-2009 se intentó implementar la estrategia COMBI en la ciudad de Matagalpa, Nicaragua, pero no se obtuvieron los resultados esperados debido al poco apoyo del gobierno nacional y municipal, asimismo, el tiempo de planificación e implementación de la metodología fue insuficiente, afectado además porque los equipos de trabajo responsables de los proyectos COMBI no eran exclusivos para la estrategia y tenían otras actividades a cargo ${ }_{(5)}$.

En Paraguay, mediante la implementación de la estrategia COMBI, se ha fortalecido la integración y la articulación del sector salud con otros sectores, especialmente con las autoridades municipales y las comisiones vecinales, el Ministerio de Ambiente, las entidades de seguridad social y las Fuerzas Armadas ${ }_{(46)}$.

En Perú se ha documentado el uso de la estrategia COMBI en brotes epidémicos de dengue en las ciudades de Iquitos en el año 2011 y Pucallpa durante el 2012, con el objetivo de promover y crear conciencia en la población sobre la importancia de la prevención y el reconocimiento de los signos y síntomas del dengue. De esta experiencia, se destaca la creación de alianzas estratégicas con diferentes actores para la difusión de mensajes para enfrentar el brote, organización de campañas que permitieron la recolección de 187 toneladas de inservibles en Iquitos y $1300 \mathrm{~m}^{3}$ de residuos sólidos en la ciudad de Pucallpa, de igual forma, se reorientaron los mensajes y acciones de comunicación de acuerdo a los escenarios epidemiológicos encontrados y las características específicas de las comunidades, identificando los mensajes clave que se debían enfatizar, entre ellos, el de la responsabilidad de toda la familia en la limpieza de posibles criaderos de vectores en la casa ya que una de las barreras para esta práctica era la creencia de que esta actividad era responsabilidad de las autoridades locales ${ }_{(52)}$.

\section{Discusión}

En los últimos años, las ETV han cobrado importancia a nivel mundial por el aumento de su incidencia en regiones endémicas y la aparición de estas enfermedades en nuevas regiones, relacionándose con modestos cambios en la estructura social y el desarrollo socioeconómico de cada región, por lo que su control exitoso requiere de una rápida identificación, acción oportuna y medidas sociales rigurosas ${ }_{(53,54)}$.

Por lo anterior, la OPS incentiva la creación de programas de prevención y control de las ETV basados en la promoción de la salud y la coordinación de acciones entre los diferentes sectores, que fomenten el cambio de conducta a nivel individual y colectivo, como la estrategia COMBI ${ }_{(55)}$.

El conocimiento de la metodología paso a paso de la estrategia de intervención COMBI es uno de los determinantes del 
éxito o no de la misma ${ }_{(37)}$ y aunque se han creado las guías para su implementación, estas se deben tomar como un documento orientador y no como una norma que se deba cumplir estrictamente, con el fin de no perder la espontaneidad y creatividad en el proceso ${ }_{(5)}$. Asimismo, es indispensable que el equipo implementador conozca y sea consciente del comportamiento a sugerir y ofrezca a las personas oportunidades frecuentes de realizar una revisión deliberada de los comportamientos sugeridos, ponderando su valor en relación con la ejecución ${ }_{(5)}$

La etapa de planificación de la estrategia COMBI es un componente fundamental que incide finalmente en el éxito o fracaso del programa, por lo que la conformación de un equipo interdisciplinario, la sistematización de la estrategia, la capacitación del talento humano y la creación de estrategias sociales, teniendo en cuenta las características etnográficas de la población, son ejes fundamentales para que las comunidades y personas puedan adoptar prácticas protectoras saludables y preventivas.

Otro determinante del éxito de la estrategia COMBI es la creación de procesos sostenibles para alentar a los individuos a mantener los cambios conductuales adoptados (23.55), estudios en comunidades demuestran la necesidad de que los programas de participación comunitaria continúen mientras la enfermedad continúe siendo una amenaza, con el fin de extender los beneficios de estas iniciativas. El no alcanzar los objetivos deseados y no satisfacer las expectativas por la falta de continuidad de estos programas, influye en la toma de decisiones por organismos gubernamentales para invertir y apoyar estas iniciativas y en consecuencia, estos programas suelen ser relegados para servir como proyectos epidemiológicos durante los brotes como el dengue.

En las evaluaciones del desarrollo de la estrategia COMBI, se ha observado que a pesar del esfuerzo realizado para su implementación aún existe predominio del enfoque vertical o transmisionista con intervenciones enfocadas en IEC, el cual se limita a la comunicación mediante visitas domiciliarias, panfletos y diferentes acciones verticales . Este comportamiento está relacionado a su vez con las desventajas desde la perspectiva económica que tiene la estrategia, debido a que estos programas son difíciles de implementar porque toman más tiempo en reflejar el impacto en comparación con un programa vertical que es de bajo costo, lo que desanima a los gobiernos en términos de financiación ${ }_{(57)}$.

Con el fin de disminuir el costo del uso de la metodología COMBI, en ocasiones se sugiere el uso de voluntarios no remunerados que difundan la estrategia ${ }_{(56)}$ como los líderes comunitarios que son indispensables para dar continuidad a los programas, debido a que son canales efectivos a través de los cuales se difunde información, se educa a la comunidad y se evalúa el cambio de comportamiento ${ }_{(5)}$. Aunque esta idea puede ser una alternativa para disminuir costos en la implementación de la estrategia, es importante incentivar a los líderes con el fin de lograr la sostenibilidad de la misma.

Conviene seguir consolidando los resultados de la implementación de la estrategia COMBI en los países donde se ha desarrollado el programa, con el fin de evidenciar los resultados positivos que permitan lograr una mayor voluntad política y la obtención de recursos económicos necesarios para apoyar y dar mayor sostenibilidad a las estrategias de control y prevención de las ETV en América Latina.

Es necesario fortalecer el monitoreo y evaluación de la implementación de la estrategia COMBI, mediante la comparación de los datos epidemiológicos y entomológicos. Adicionalmente, debe existir un compromiso político administrativo para llevar a cabo la implementación y sostenibilidad de la estrategia COMBI con el fin de obtener los resultados esperados.

\section{Agradecimientos}

A los Training Programs in Epidemiology and Public Health Interventions Network TEPHINET, ya que esta revisión se realizó en el marco de un minigrant financiado por dicha institución. 


\section{Referencias}

1. OMS. Nota descriptiva $\mathrm{N}^{\circ}$ 387. Enfermedades Transmitidas por Vectores [Internet] 2016 [consultado 2016 May 30] Disponible en: http://www.who.int/mediacentre/factsheets/ fs $387 / \mathrm{es} /$

2. Walker K, Lynch M. Contributions of Anopheles larval control to malaria suppression in tropical Africa: review of achievements and potential. Med Vet Entomol. 2007;21:p.221

3. Rinker D., Pitts J., Zwiebel L. Disease vectors in the era of next generation sequencing. Genome Biol. 2016; 17:p. 95.

4. WHO Mediterranean Centre for Vulnerability Reduction (WMC). Mobilizing for action Communication for Behavioural Impact (COMBI). Tunis, Tunisia, 2004.

5. OPS. Sistematización de lecciones aprendidas en proyectos COMBI en Dengue en América Latina 2011

6. OMS., OPS. Comunicación para impactar en conducta (COMBI). Disponible en: http://www. paho.org/hq/index.php?option=com_content\&v ie $w=\operatorname{article} \& i d=4504 \% 3 \mathrm{~A} 2010-\mathrm{comunic}$ acionimpactar-conducta-combi\&catid $=901 \% 3$ Adenguecontent\&Itemid $=41040 \&$ lang $=$ es

7. Hosein E. COMBI: Communication for Behavioural Impact. [consultado 2016 May 30] Disponible: http://www.comminit. $\mathrm{com} /$ content/combi-communication-behavioural-impact.

8. OPS. Resolución CD 43. R4 Dengue y dengue hemorrágico. Washington, DC, OPS. [Internet] 2001 [consultado 2016 May 30] Disponible en: http://www.paho.org/per/index. php?option $=$ com_docman $\&$ view $=$ document $\&$ category slug $=$ dengue $-000 \&$ alias $=267-\mathrm{cd} 43-\mathrm{r} 4-2001$-resoluciondengue-dengue-hemorragico-7\&Itemid=1031

9. OMS. 55 a Asamblea Mundial de la Salud. Prevención y control de la fiebre dengue y la fiebre hemorrágica dengue. [Internet] 2002. [consultado 2017 May 30] Disponible: http:// apps.who.int/iris/handle/10665/81996.

10.OPS. Resolución CD 44.14 Dengue. Washington, DC, OPS. [Internet] 2003 [consultado 2017 May 30] Disponible en: http://www.paho.org/per.../index.php?option=com_do $\mathrm{cman} \&$ view $=$ document $\&$ layout $=$ default $\&$ alias $=269-\mathrm{cd} 44-$ 14 -setiembre-2003-dengue-9\&category_slug=dengue$000 \&$ format $=$ html\&Itemid $=1031$.

11.-OPS. Resolución CD 44.R9. Washington, DC, OPS. [Internet] 2003 [consultado 2017 May 30]. Disponible en: http://www.paho.org/spanish/gov/cd/cd44-r9-s.pdf.
12. Ministerio de Salud de Protección Social. Guía para el diseño, implementación, monitoreo y evaluación de la estrategia de Abogacía, Comunicación y Movilización Social (ACSM) para el Programa de Tuberculosis. 2012.

13. Shepherd J., Kavanagh J., Picot J., Cooper K., Harden A., Barnett-Page E., Jones J., Clegg A., Hartwell D., Frampton GK., and Price A. The effectiveness and costeffectiveness of behavioural interventions for the prevention of sexually transmitted infections in young people aged 13-19: a systematic review and economic evaluation. Health Technology Assessment. 2010; 14 (7)

14. Bertrand J T, O'Reilly K, Denison J, Anhang R, Sweat M. Systematic review of the effectiveness of mass communication programs to change HIV/AIDS-related behaviors in developing countries. Health Education Research 2006; 21(4): p. 567-597.

15. Robin L, Dittus P, Whitaker D, Crosby R, Ethier K, Mezoff J, Miller K, Pappas-Deluca K. Behavioral interventions to reduce incidence of HIV, STD, and pregnancy among adolescents: a decade in review. Journal of Adolescent Health 2004; 34(1): p.3-26.

16. Do HT., Santos JA., Trieu K., Petersen K., Le MB., Lai DT., Bauman A., Webster J. Effectiveness of a Communication for Behavioral Impact (COMBI) Intervention to Reduce Salt Intake in a Vietnamese Province Based on Estimations From Spot Urine Samples. J Clin Hypertens (Greenwich). 2016 Nov;18(11):p.1135-1142.

17. Kang-I C. Communication for behavioral impact: a COMBI plan for cancer prevention in Taiwan. University Graduate School. December, 2014.

18. Parks W., Lloyd L. Planificación de la movilización y comunicación social para la prevención y control del dengue. Guía paso a paso. 2004

19. OMS, OPS, UNICEF. Communication for behavioural impact (COMBI). A toolkit for behavioural and social communication in outbreak response.

20.Social Mobilization and Social Marketing in Developing Countries: Lessons for Communicators. Penang: Southbound. 1992

21. Ministerio de Salud y Protección Social. Comunicación y movilización social para la prevención y control de la malaria en Colombia. Marzo 2015.

22Fritzell C, Raude J, Adde A, Dusfour I, Quenel P, Flamand C (2016) Knowledge, Attitude and Practices of Vector-Borne Disease Prevention during the Emergence of a New Arbovirus: 
Implications for the Control of Chikungunya Virus in French Guiana. PLoS Negl Trop Dis 10(11).

23. WHO. Global Strategy For Dengue Prevention And Control 2012-2020. Año 2012

24. Susanta K Ghosh, Rajan R Patil, Satyanarayan Tiwari and Aditya P Dash. A community-based health education programme for bio-environmental control of malaria through folk theatre (Kalajatha) in rural India. Malaria Journal.2006, 5:p.123

25. Alcaldia de Monteria, ESE CAMU El Amparo, proyecto "acompañamiento en la implementación de la metodología "COMBI 10 pasos" para la identificación e intervención de conductas de riesgo a nivel individual, familiar, comunitario e institucional, que adelanta la E.S.E. CAMU El Amparo". Disponible en: http://monteria.gov.co/docs/2015/combi.pdf

26. Escudero-Támara E, Villareal-Amaris G. Intervención educativa para el control del dengue en entornos familiares en una comunidad de Colombia. Rev. Perú Med. Exp. Salud Pública. 2015;32(1):p.19-25.

27. OMS., OPS. Evaluación para el fortalecimiento de procesos de participación social en la promoción y el desarrollo de la salud en los sistemas locales de salud. Washington D.C, 1994

28. Comisión Nacional de Seguimiento a la Propuesta de Reforma Integral de Salud. Propuesta política de participación social en salud. El Salvador. Mayo 2006.

29. Sanabria G. Participación social en el campo de la salud. Rev. Cubana Salud Pública. 2004;30(3)

30. OMS. Promoción de Salud. Glosario. Ginebra, 1998.

31. Wallerstein N. Power betwen evaluator and community: research relationships within New Mexicos's healthier communities. Social Science of Medicine. 1999;49(1):p.39-53

32.Déclaration de Jakarta sur la promotion de la santé au XXI siecle. OMS; Ministere de la Santé, Jakarta: Republique de L’Indonésie, 1997.

33. WHO. Carta de Bangkok para la promoción de la salud en un mundo globalizado. Disponible en: www.who.int/ healthpromotion/conferences/6gchp/BCHP_es.pdf

34. Zamudio M. Teoría y guía práctica para la promoción de la salud. Québec: Universidad de Montreal; 1998.

35. OMS. Carta de Ottawa para la Promoción de la Salud. Disponible en: www.promocion.salud.gob.mx/dgps/ descargas 1/promocion/2_carta_de_ottawa.pdf
36. Avila G. Martínez M., Ponce C., Ponce E., Rosales R., Orellana L., Quintana M. Participación Comunitaria para el Control de la Enfermedad de Chagas: Experiencia en una área Endémica de Honduras. Revista Medica Hondureña .1996; 64 (2)

37. Tapia-Conyer R., Mendez-Galván J, Burciaga-Zuñiga P. Community participation in the prevention and control of dengue: the patio limpio strategy in Mexico. Paediatrics and International Child Health .2012; 32 (1)

38. Andersson N., Nava-Aguilera E., Arosteguí J., MoralesPerez A., Suazo-Laguna H., Legorreta-Soberanis J, HernandezAlvarez C., Fernandez-Salas I., Paredes-Solís S., Balmaseda A., Cortés-Guzmán A, Serrano de los Santos R., Coloma J, Ledogar R. Harris E. Evidence based community mobilization for dengue prevention in Nicaragua and Mexico (Camino Verde, the Green Way): cluster randomized controlled trial. BMJ . 2015; 351 (3):p.267

39. Sánchez L, Pérez D, Alfonso L, Castro M, Sánchez LM, Van der Stuyft P, et al. Estrategia de educación popular para promover la participación comunitaria en la prevención del dengue en Cuba. Rev. Panam Salud Publica. 2008;24(1):p.619.

40. Fernández E, Martínez M, Sherman C. Social Mobilization for Dengue Control in Honduras. Dengue Bulletin. 2004; 28.

41. Baly A, Toledo ME, Vanlerberghe V, Ceballos E, Reyes A, Sanchez I, et. al. Cost-effectiveness of a community- based approach intertwined with a vertical Aedes control program. Am J Trop Med Hyg.2009;81:p.88-93.

42. Vanlerberghe V, Toledo ME, Rodríguez M et al.Community involvement in dengue vector control: cluster randomized trial. British Medical Journal. 2009; 338, b1959.

43. Capraraa A., Wellington J., Rocha Peixotoa A., Monteiro C., Soares Nobrea J., Sommerfeldb J., Kroeger A. Entomological impact and social participation in dengue control: a cluster randomized trial in Fortaleza, Brazil. Trans R Soc Trop Med Hyg. 2015; 109: p.99-105

44. Liborio M, Tomisami A, Moyano C, Salazar R \& Balparda L (2004) Estrategias de prevención de dengue-Rosario, Argentina. Revista Brasileira de Epidemiologia; 7: p.311-327.

45. Elder J. Community involvement in dengue vector control. BMJ. 2009;338:p.1023

46. San Martín J., Brathwaite-Dick O. La Estrategia de Gestión Integrada para la Prevención y el Control del Dengue en América Latina Rev. Panam Salud Publica/Pan Am J Public Health. 2007; 21(1) 
47. Universidad de Antioquia. Informe de resultados del desarrollo de la metodología COMBI - malaria en los departamentos de Antioquia, Cauca, Chocó, Córdoba y Valle del Cauca, 2010 - 2014. Proyecto Malaria ColombiaComponente Social.

48. Mosquera M., Obregón R., Lloyd L. Orozco M., Peña A. Comunicación, movilización y participación: lecciones aprendidas en la prevención y control de la fiebre dengue. Rev. Investigación y desarrollo. 2006; 14 (1): p. 120-151

49. Escudero-Támara E, Villareal-Amaris G. Intervención educativa para el control del dengue en entornos familiares en una comunidad de Colombia. Rev. Perú Med Exp Salud Publica. 2015;32(1):p.19-25.

50. Universidad de Antioquia. Seminario nacional experiencias en comunicación y movilización para el impacto conductual (COMBI) en Colombia. Proyecto Malaria Colombia.

51. Ministerio de Salud Pública. Alcance al informe sobre la ejecución del plan COMBI - Cooperativa Guayas y Quil II Guasmo Sur periodo agosto - diciembre 2013. Comunicación social. Enero 2014

52. OMS-OPS. Respuesta a los brotes de dengue en las ciudades de Pucallpa e Iquitos, Perú. Documento de sistematización. 2013

53. Kilpatrick A., Randolph S. Drivers, dynamics, and control of emerging vector-borne zoonotic diseases. Lancet. 2012; 380(9857):p. 1946-1955.

54. Setbon M., Raude J. Population response to the risk of vector-borne diseases: lessons learned from socio-behavioural research during large-scale outbreaks. Emerging Health Threats Journal 2009.

55.OMS, OPS. Estrategia de Gestión Integrada de prevención y control de dengue para los Estados Partes y Asociados del MERCOSUR. 28 de mayo al 2 de junio de 2017.

56.Vega-Casanova J., Vega-Estarita J., Arroyave-Cabrera J. Lecciones aprendidas en la comunicación en salud y de riesgo en el manejo del virus del Chikungunya y otras enfermedades transmitidas por el mismo vector. Rev. Salud Uninorte. 2016; 32 (1):p. 35-55

57. Baly A, Toledo ME, Boalaert M, Reyes A, Vanlerberghe V, Ceballos E., et al. Cost effectiveness of Aedes aegypti control programmes: participatory versus vertical. Transactions of the Royal Society of Tropical Medicine and Hygiene 2007; 101 (6): 578-586. DOI: 10.1016/j.trstmh.2007.01.002. 